\title{
Practical Consideration Factors to Design Array Configuration of Direction Finding System for Airborne Signal Intelligence
}

\author{
Jong-Hwan Lee, Jeung-Min Joo, Kichul Kim, Jung-Hoon Lee $\mathbb{D}$, and Young-Ju Park \\ Agency for Defense Development, Electronic Warfare PEO, Daejeon, Republic of Korea \\ Correspondence should be addressed to Jung-Hoon Lee; yougoal@naver.com
}

Received 16 November 2017; Revised 4 April 2018; Accepted 8 April 2018; Published 17 May 2018

Academic Editor: Kiseon Kim

Copyright (C) 2018 Jong-Hwan Lee et al. This is an open access article distributed under the Creative Commons Attribution License, which permits unrestricted use, distribution, and reproduction in any medium, provided the original work is properly cited.

\begin{abstract}
Airborne signal intelligence (SIGINT) systems must be capable of locating radio signal sources. Direction finding (DF) to support this capability is an important factor. There are some practical considerations to be taken when designing the array configuration of a DF system for airborne SIGINT systems. This paper summarizes the practical factors when designing the array configuration of the DF system for airborne SIGINT. In particular, it focuses on four areas: antenna consideration factors when installing the DF system for airborne SIGINT from a practical point of view, array configuration methods for airborne communications intelligence and electronic intelligence, and a numerical analysis to select the optimum antenna position for airborne SIGINT.
\end{abstract}

\section{Introduction}

An aircraft signal intelligence (SIGINT) system is used strategically and tactically to gather signals from radar and communications systems. It is commonly divided into communications intelligence (COMINT) and electronic intelligence (ELINT) systems. Because SIGINT systems require the ability to locate the source of enemy signals [1], direction finding (DF) to support this capability is an important requirement and basically operates in a wide frequency range from $20 \mathrm{MHz}$ to $40 \mathrm{GHz}$ [2]. To perform DF over this wide frequency range and a wide field of view (FOV), airborne SIGINT uses several antennas according to the frequency range. For example, ELINT generally uses a spiral type of antenna [3] and COMINT uses a blade type. In addition, there are several limitations when installing a DF system for airborne SIGINT related to the limited space available for antenna arrays and the effect of reflection from aircraft structures [4]. These may degrade the DF accuracy.

This paper summarizes the practical factors involved when designing the antenna array configuration of the DF system for airborne SIGINT. The outline of this paper is as follows. Section 2 briefly mentions the factors to consider when selecting airborne SIGINT antennas. This section does not focus on the design or performance of the antenna itself but rather on the factors required in the selection of the antenna used in configuring the antenna array for a SIGINT system. Section 3 presents the practical factors to consider when designing the DF array configuration for airborne COMINT. First, the received signal model is introduced. The design and evaluation criteria for the DF system are briefly described. The DF algorithms and array configuration are presented.

Finally, calibration methods are described. Section 4 presents the necessary factors to consider for the DF array configuration for airborne ELINT. Thus, this section focuses on the array spacing without angle ambiguity, the DF accuracy of an interferometer DF system, some methods for obtaining multiple element arrays, and the probability of ambiguity based on the signal to ratio (SNR) and array configuration type for a three-element array. Finally, it describes the computational electromagnetic (CEM) modeling and simulation needed to accurately estimate the performance of the DF antenna array. The antennas in the array can have very different kinds of impedance and radiation patterns because of their different installation positions and electromagnetic environments on a platform. Several CEM modeling methods are introduced, and the recently developed AntOpt software, which is based on a uniform geometrical theory of diffraction (UTD), is briefly discussed in this section. It is shown that the 
computation results obtained from AntOpt agree with those from FEKO, which is based on a moment method and widely used in computational electromagnetic studies.

\section{Factors to Consider for Airborne SIGINT Antennas from Practical Point of View}

For airborne SIGINT operations, signals with a wide frequency spectrum and unknown direction of arrival (DOA) must be received by an antenna that can be mounted in the aircraft. Thus, the general requirements for an airborne SIGINT antenna are a miniaturized volume, planar configuration, relatively wide frequency range, appropriate antenna pattern, and suitable physical shape for aviation operation. In other words, the physical, electrical, and environmental characteristics must be checked before applying the airborne antenna. There are several kinds of antennas for SIGINT use that can be installed in an aircraft. In a practical sense, in the case of a relatively low frequency band, wire-type and blade antennas can be used, while spiral, horn, patch, and aperture or slot antennas can be employed in the middle and high frequency bands [5]. In some cases, a corner or parabolic reflector with an appropriate feeder has been applied as the main antenna when high gain (directivity) is required for long-range or satellite communication. Except for the special case of an antenna installed on the top of the vertical fin of the aircraft, the antennas are usually installed on the skin of the upper or lower fuselage of the aircraft. With this installation environment, this paper considers three factors in antenna selection: physical, electrical, and environmental aspects.

2.1. Consideration of Physical Characteristics. Unlike the case of an antenna installation at a ground station, restrictions on the volume, weight, and configuration of the antenna are strictly applied in the case of an airborne system. In general, for a frequency range of a few megahertz to tens of megahertz, a wire-type antenna is conventionally used [6]. However, the estimated wire length is supposed to be too long for installation outside an aircraft without changing or degrading the performance of the antenna. Thus, the use of this type of antenna is limited, even though there are instances where it is used. When high-power transmission is intended, a waveguide slot antenna can generally be used [7]. However, because high-power transmission is of no concern, a waveguide slot antenna will be excluded at this time. An antenna with a reflector also has high gain characteristics, but it is undesirable because it requires a large installation volume and curved surface radome. In addition, it is not adequate for use as the single antenna of an interferometer array.

Therefore, an airborne antenna is usually confined to a type that can be relatively miniaturized compared with the ordinary volume or one with a plane radiation aperture. This means that the blade, spiral, and horn antennas become the preferred antennas based on the physical aspect. These types of antennas are relatively easy to mount on the skin of an aircraft. Thus, it can be concluded that the shape and form of the radiation plane are the most important factors in the physical domain.
2.2. Consideration of Electrical Characteristics. After the physical characteristics, it is necessary to consider the electrical characteristics such as the frequency range, pattern, and gain. First, wide band characteristics are required. Generally, the signals from threats distributed over a very wide RF spectrum must be received and processed by a single system. Thus, the antennas of the system must cover that frequency range. In most SIGINT systems, the frequency band is often divided into multiple bands [8]. Although the operating band is separated into several bands, the antenna must still have a multiple octave bandwidth. While the spiral [9] and horn antennas satisfy this criterion, a conventional monopole or any wire-type antenna is unsuitable from this standpoint. However, some airborne blade antennas [10-12] cover a frequency range of tens of megahertz to hundreds of megahertz or hundreds of megahertz to a few gigahertz, although they can have a low gain at a low frequency band. The pattern must also be considered. To receive signals from arbitrary directions, the antenna must have a wide beamwidth, which means that the FOV of the antenna has to show an omnidirectional pattern or moderate pattern curve. The pattern of a blade antenna is basically omnidirectional, and a spiral or horn shows a smooth pattern curve in the FOV. A constant gain cannot be maintained over the whole frequency band even in the case of a wide band antenna. In general, the gain is lower in a relatively low frequency range, while a higher gain can be obtained in a relatively high frequency range. For example, a wide band antenna often shows a gain with a negative $\mathrm{dBi}$ value in the low frequency band, while a gain with a positive dBi value appears in the high frequency band [5]. The system is designed so that the $\mathrm{RF} /$ digital process boards of the receivers are more sensitive or the length of the cable connecting the antenna and receiver is strictly limited.

The polarization of the threats is also a factor to be considered. Because the communications emitters in the COMINT band generally have a vertical polarization and the radars in the ELINT band operate with both vertical and horizontal polarization [8], a blade antenna for the COMINT band and spiral for the ELINT band are preferred and generally used in the system.

2.3. Consideration of Environmental Characteristics. Finally, an investigation of the environmental characteristics must be performed. In fact, once the physical characteristics and electrical performance of the antenna are determined, the matter of the environmental characteristics can be solved by simply selecting one of the antennas that satisfy the environmental specifications or requesting that the vendor customizes an existing antenna to make it suitable for the environmental requirements. In most cases, the former is done. When an adequate antenna for the required environmental specifications is being selected, the factors that must be considered include the temperature, humidity, altitude, vibration, and shock [13]. Basically, the military standards [14] for the environmental requirements are applied to a product used in a military system, although other standards [15] can be applied in some cases, especially for a nonmilitary platform (aircraft). This is because the aircraft used as the 
platform is a civilian model in this case. The standard documents describe the method and procedure according to the platform, usage, and conditions under which the product is used. The specification values, duration, and type of test cycle are also documented there.

In the case of a SIGINT system, these values and other factors must be chosen according to the category for the SIGINT system installed in a civilian aircraft platform for military use. Then, the antenna can be selected from among several antennas that satisfy these criteria. For example, a blade antenna with a low temperature limit of $-54^{\circ} \mathrm{C}$ can be selected according to the military standard (method 501.3, proc. I \& II) for an antenna mounted on the skin of an aircraft.

\subsection{Systemic Approach for Antenna Selection. In addition to} the previously mentioned criteria, a systemic approach is needed for the antenna installation. In the frequency range corresponding to the COMINT band, the use of a spiral or horn can be very restricted because the resultant antenna is supposed to be too large to be used. Moreover, there are existing blade antennas that cover the COMINT frequency range and have a nearly omnidirectional pattern [16]. Thus, a blade antenna is frequently used as a COMINT antenna. On the other hand, in the ELINT band, the spiral and horn antennas are frequently used for the system because they have appropriate physical volumes and performances (moderate gain, adequate pattern, and so on) [17]. Therefore, it can be concluded that when the interferometer array is configured, the blade antenna could be profitably used in the array for COMINT and the spiral or horn antenna would be advantageous in the array for ELINT. For this reason, as the airborne antennas, the blade antenna is selected for the COMINT operation and the spiral or horn antenna is employed for the ELINT operation.

\section{Facts to Consider for Antenna Array Configuration for Airborne COMINT}

3.1. Signal Model and Practical Considerations. Consider a general array configuration with $N$ antenna elements. The response of such an array for $M$ impinging signals under ideal circumstances is described. Isotropic, mutually independent antenna elements are assumed. It is also assumed that the source signals are narrowband signals and the modulation is constant during the propagation delay for any antenna element. Let us consider the $k$ th source signal, coming from azimuth and elevation $\left(\alpha_{k}, \beta_{k}\right)$, with carrier frequency $f_{0}$ and complex modulation function $S_{k}(t)$. Then, the received signals of all $N$ antenna elements can be modeled as follows [18]:

$$
\begin{aligned}
Z(t)= & \sum_{k=1}^{K} S_{k}(t)\left(\begin{array}{c}
\exp \left(j 2 \pi f_{0}\left(t-\tau_{1}\left(\alpha_{k}, \beta_{k}\right)\right)\right) \\
\vdots \\
\exp \left(j 2 \pi f_{0}\left(t-\tau_{N}\left(\alpha_{k}, \beta_{k}\right)\right)\right)
\end{array}\right) \\
& +W(t)
\end{aligned}
$$

where $W(t)$ represents the background noise of all the antenna elements and the noise components of all the elements are independent. $\tau_{i}\left(\alpha_{k}, \beta_{k}\right)$ accounts for the propagation delay of the $i$ th antenna element and $k$ th source signal. The number of antenna elements is $N$. Although designing an isotropic, point-like, mutually independent antenna array is never realized in a practical DF system, the simple expression that is just introduced can be used to obtain the expected performance of an antenna array. Because we assumed isotropic elements, the array pattern is completely determined by the spatial arrangement. If the elements are still independent and there is no mutual coupling, the array pattern can be computed simply by multiplying a single element's pattern at the origin by the array factor that accounts for the array configuration [19].

In the general case, however, the array factor and a single element's pattern are inseparable, because we have to consider the distortions of the antenna patterns that stem from the DF components and DF environment. Some of the practical effects are as follows. First, a misplacement of the antenna positions, which is predictable when installing the antennas, might cause a distortion of the antenna pattern. Second, the tolerance of the phase accuracy of the antenna cables and frequency stability of the RF front-ends are factors that aggravate the designed antenna pattern. The third effect is the mutual coupling between antenna elements. This becomes prominent at high frequencies where elements have to be grouped close together. The fourth effect is the interdependency of the antenna elements and DF platform. The distance between the elements and platform is too close to neglect its influence on the antenna pattern. The fifth effect is the reflection caused by obstacles on the DF platform, which means that the antenna pattern is the result of a combination of interfering signal components. Finally, the diffraction caused by obstacles on the DF platform and the platform itself may be one of the causes of distorted antenna patterns. Accordingly, the array pattern cannot be simply evaluated in practice but has to be discretized with respect to the frequency, azimuth, and elevation on a predefined grid.

3.2. Design Criteria. When designing broadband DF arrays, there are typically two competing requirements. On the one hand, a narrow width for the main beam of an array pattern is desirable to achieve a high DF accuracy [20]. This can be achieved by designing the largest possible apertures, namely, large element spacing. On the other hand, a large bandwidth for the array is of practical interest, in order to achieve a good coverage of the frequency range [21]. As the frequency increases, however, the relative spacing of the antenna elements in terms of the wavelength becomes larger and the grating lobes increase, which degrades the DF performance. Hence, a tradeoff between a reasonable DF accuracy and bandwidth is inevitable. For desirable array configurations, design criteria such as the main beamwidth, bandwidth, and directional gain have to be considered. It should also be noted that the final array configuration may deviate from the initial one because of installation restrictions on the DF platform or undesirable disturbance from obstacles mounted on the platform. 
In summary, the following evaluation criteria should be considered in order to quantify the design criteria: the halfpower beamwidth of the main beam, side lobe distance, directional gain with respect to an isotropic antenna element, RMS performance for DF accuracy, and bandwidth.

\subsection{DF Algorithms and Array Configuration. Dense signal} scenarios are unavoidable in airborne COMINT applications because of the extended radio horizon. Furthermore, a significant portion of the observed spectrum contains various signals on the same frequency coming from multiple directions. This is why scarce frequency resources on the ground are reused at adequate spatial distances. Classical DF algorithms are designed to detect one signal and deliver DF results only for the strongest signal component in the case of multiwave incidence. However, the DF results in multiwave incidence may be distorted and occasionally provide the wrong results. The interferometer and beamforming approaches are examples of classical DF algorithms. Super-resolution algorithms evaluate the DF results for each component of a signal mixture, where the components may overlap completely in time and frequency [22]. The estimate of the signal number represents an integral part of any superresolution DF algorithm. The separation into different signal components can improve the accuracy of the DF results and subsequent position fixing of the signals.

Moreover, super-resolution algorithms can provide the essential feature of obtaining DF results for weak signal components that have strong signal components superimposed over them. The DF performance is also critical for the array constellation, as well as the DF algorithm. For general array configurations, the following should be considered when designing an antenna array. (1) Sensitivity: Because weak signals are of specific interest, the minimum required receive level for a successful DF estimation is determined. (2) Bandwidth: As previously mentioned, a tradeoff between the array bandwidth and DF performance is inevitable. (3) Signal separation capability: This is strongly related to the number of antenna elements, as well as a given array beamwidth. (4) Signal correlation: Large correlation values between signal components degrade the DF performance. The ideal is a correlation value of zero. In practical terms, however, the correlation values for the received signals might not be zero. (5) RMS performance: In general, this criterion is the most important reference point for the DF accuracy. (6) Wild bearing percentage: The percentage of outliers is used as a reliability measure for the DF quality. In other words, the azimuth result with a deviation of more than a certain value from the true direction is defined as a wild bearing.

The array configuration is very important for the performance of DF systems, and some examples have been investigated $[6,7,16]$. In designing the array configuration, two or three bands are typically partitioned to cover a wide frequency range of interest. The low frequency band array design might be based on a V-shape or Y-shape in order to obtain reasonably large antenna apertures. All of the elements are assumed to be mounted on the fuselage, possibly on a ground plane. A better aperture in the vertical dimension can be accomplished by mounting antenna elements on the wings. However, this may cause difficulties with shadowing effects from the body and fluttering of the plane's wings. The high frequency band array design may be based on a uniform circular array approach. An alternative could be a closer, diamond-shaped arrangement of the antenna elements if the space is not available for a uniform circular array of this size.

3.4. Platform Calibration. Mounting the antenna array on an airborne platform may result in significant changes in the antenna pattern due to the environment around the array. For example, various obstacles around the array such as metal objects will change the beam pattern [23]. Diffraction and reflections may change the course of the phase fronts [24]. The need for calibration measures on airborne platforms in general is well known. Therefore, platform calibration is one of the most important tasks when integrating an antenna array. The goal of platform calibration measurements is to collect knowledge of the signal received by the sensor array in an electromagnetic field that is as complete and precise as possible.

Recently, the availability of superior simulation methods seems to have enabled the accurate simulation of the platform installed performance of an antenna. However, for more accurate results and better DF performance, the platform calibration has been taken into account. The platform calibration can be categorized mainly as a mock-up calibration and an inflight calibration. Mock-up calibration data are measured in a measurement chamber or using groundbased field measurements. The antenna arrays are installed on a mock-up. Mock-up constructions can cover a wide variety of physical realizations. The mock-up may only approximate the real platform in some respect to qualitatively demonstrate characteristic features. It can also be intended to be a copy of the real platform that is exact as possible. According to the degree of similarity between the actual platform and the mock-up, various phenomena observed in inflight measurement can be explained and understood. The calibration data obtained by inflight measurements are more accurate and practical than ground-based mock-up-based measurements. The mock-up-based calibration data cannot include unexpected phenomena caused by small obstacles mounted on an airborne aircraft, and inflight propagation conditions are difficult to achieve with real-size mock-up measurements. This is because the mock-up is relatively close to the ground, which allows the signals to be influenced by various objects around it on the ground. The amount of frequency and azimuth characteristics of the received signals obtained in an actual flight is significantly smaller than the coverage in ground-based measurements.

The actual flight is affected by the constraints of the maximum transmission signal strength, the frequency permissions by the telecommunications regulatory authority, and the ever-changing spectral environment. Depending on the weather conditions, the plane's attitude may be severely shaken, thus preventing the desired data from being obtained. Well-defined and constant measurement conditions such as a constant SNR during the measurement cannot be established for inflight tests. 


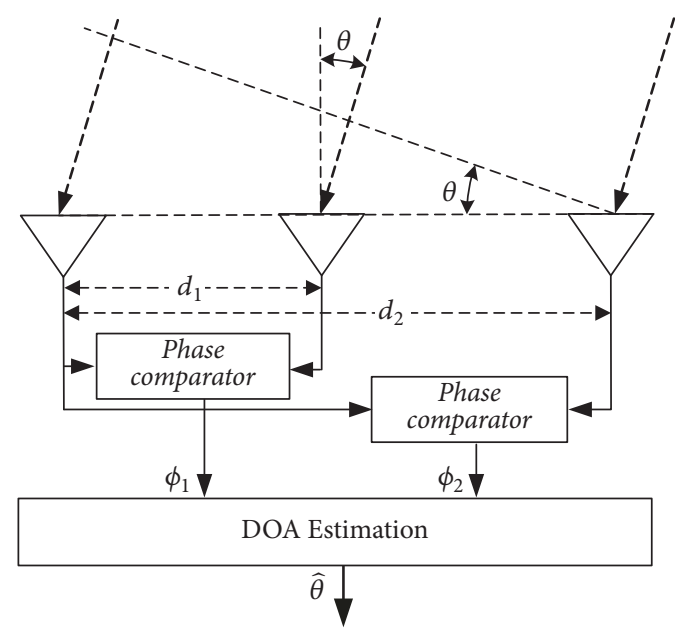

(a)

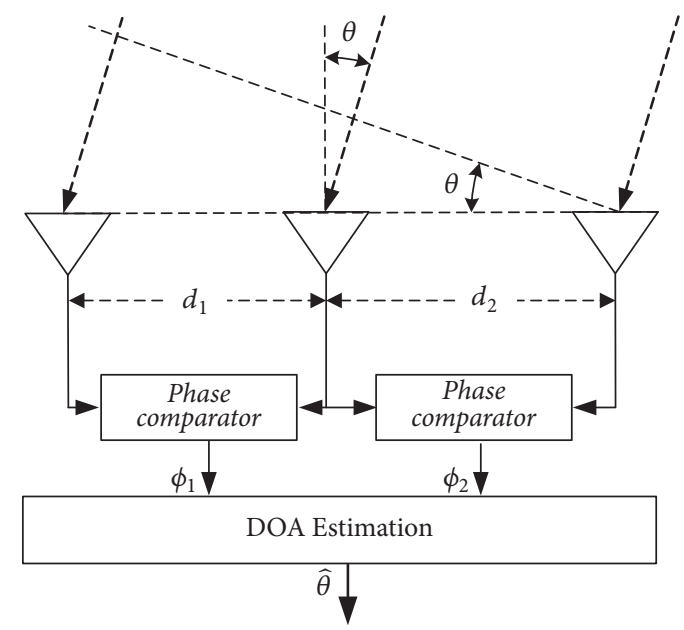

(b)

FIgURE 1: Array configuration for three-element interferometer: (a) end phase (left) and (b) midphase.

Hence, additional effort is necessary to exclude impaired data and include the desirable data. All DF measurements should be repeated to distinguish random effects from stable phenomena. Inflight calibration is much more complex, timeconsuming, and error-prone than mock-up-based calibration. It should be noted that both inflight calibration and mock-up-based calibration are important to maintain the essential DF performance.

\section{Antenna Array Configuration for Airborne ELINT}

4.1. Array Configuration for Interferometer DF System with Three-Element Array. Airborne ELINT uses a belly radome housing or pod type attached to the aircraft body to collect the radio signals that can be used for direction finding.

The interferometer DF systems for ELINT are mainly composed of a three- or four-element array according to the required degree of DF accuracy. Array configurations for a three-element interferometer with two different baselines are shown in Figure 1. The arrays may be classified according to the channel used for phase reference [25]. If the first channel is the reference for the other channel, the array is called endphase left, as shown in Figure 1(a). Similarly, if the last channel is the reference for the other channel, the array is called endphase right. Finally, if the middle channel is the reference for the other channel, the array is called midphase, as seen in Figure 1(b). Interferometer DF systems generally use multiple baselines to achieve a high DF accuracy and resolve angular ambiguity. As shown in Figure 1, the widest baseline, $d_{2}$, is used to achieve DF accuracy, and the other baseline, $d_{1}$, is used to resolve the angular ambiguity.

When a signal of wavelength $\lambda$ arrives at an angle $\theta$ relative to the array, the phase difference between two elements with a spacing $d_{i}$ obtained by the modulus operation is expressed as

$$
\phi_{i}=\psi_{i} \bmod 2 \pi, \quad i=1,2,
$$

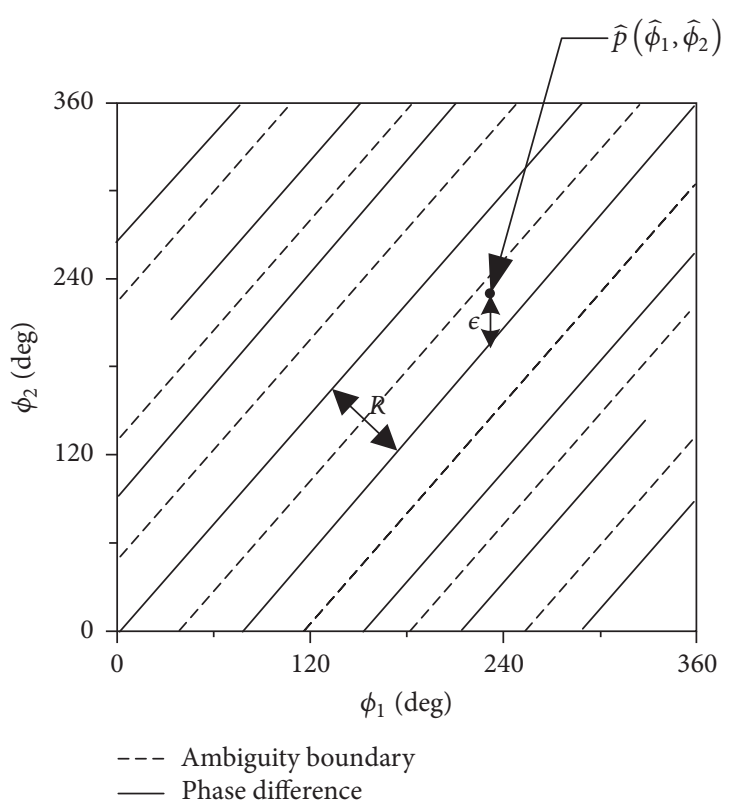

FIGURE 2: Phase sample space for three-element interferometer.

where $\psi_{i}=\left(2 \pi d_{i} / \lambda\right) \sin (\theta), 0 \leq \phi_{i}<2 \pi$. The phase sample space found using (2) is illustrated in Figure 2. Each line represents the relationship of $\phi_{1}$ and $\phi_{2}$, and $S$ is the distance between the lines of the phase difference. If $\widehat{\phi}_{1}$ and $\widehat{\phi}_{2}$ are the measured values with error term $\epsilon$ for $\widehat{\phi}_{1}$ and $\widehat{\phi}_{2}$, these values correspond to a point $\widehat{p}\left(\widehat{\phi}_{1}, \widehat{\phi}_{2}\right)$ in the sample space. In the absence of measurement error $(\epsilon=0)$, this point $\hat{p}$ would be located on one of the phase difference lines. However, $\widehat{p}$ will not lie on any of the lines in the presence of a measurement error. Moreover, if the measurement error is larger than the ambiguity boundary, the DF error may be increased and the DF performance is degraded. Hence, the optimum condition 


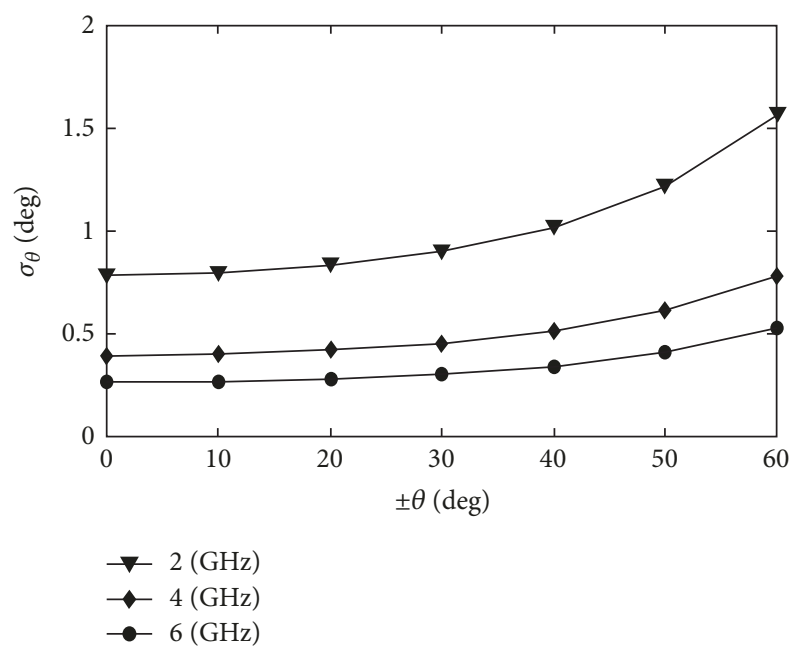

FIGURE 3: DF accuracy of three-element interferometer.

for resolving the ambiguity is for each line of the phase sample space to be as widely spaced as possible [26].

4.2. DF Accuracy for Interferometer DF System. In order to satisfy the requirement, one important factor is the DF accuracy. The theoretical root-mean-square (RMS) angular errors in terms of the SNR [27-29] are given as follows:

$$
\sigma_{\theta}=\frac{\lambda}{2 \pi d_{2} \cos (\theta) \sqrt{\mathrm{SNR}}} .
$$

In (3), the DF accuracy for a three-element interferometer with two different baselines is determined by the widest baseline. In addition, the interferometer DF system for airborne ELINT performs direction finding over a wide range of frequencies with a single array configuration. To compute the DF accuracy of the three-element array configuration in the frequency range of $2-6 \mathrm{GHz}$ with $|\theta| \leq \pi / 3$, the array spacings are $d_{1}=1 \lambda_{6}$ and $d_{2}=3.5 \lambda_{6}$ (where $\lambda_{6}$ is a wavelength of $6 \mathrm{GHz}$ ) and the SNR is $20 \mathrm{~dB}$. The DF accuracy using (3) is shown in Figure 3. As shown in Figure 3, the DF accuracy improves when the frequency increases or angle $\theta$ approaches zero.

4.3. Methods for Obtaining Multiple Element Arrays for Interferometer DF System. Various methods are used to obtain the spacing of multiple element arrays for an interferometer DF system. Two choices for the antenna element locations are available: harmonic binary spacing of $2^{n} \cdot \lambda / 2$, where $n=0,1,2,3$, or nonharmonic spacing [17]. Harmonic binary spacing can be simply selected, but the phase error as a function of the SNR has not been given. Nonharmonic binary spacing generally uses the mutually prime integer. McAulay [27] gave the ratio of the three-element array spacing as follows:

$$
\frac{m}{n}=\frac{d_{2}}{d_{1}},
$$

where $m$ and $n$ are integers and this ratio is set to be an integer. This method provides the phase error for the three-element
TABLE 1: Sample antenna array spacings for three element array.

\begin{tabular}{lccc}
\hline \multirow{2}{*}{ Array spacing type } & \multicolumn{3}{c}{ Array spacing $d_{1}, d_{2}(\lambda)$} \\
& $\operatorname{Ref}[22]$ & $\operatorname{Ref}[20]^{*}$ & $\operatorname{Ref}[18]$ \\
\hline Harmonic & $0.5,1.0$ & $0.5,1.0$ & $0.5,1.0$ \\
\multirow{2}{*}{ Nonharmonic } & - & $0.5,1.5$ & $0.5,1.5$ \\
& - & $1.0,1.5$ & - \\
& - & $0.5,2.5$ & $0.5,2.5$ \\
\hline
\end{tabular}

${ }^{*}$ Midphase.

array. Another method to obtain the three-element array spacing was provided by Goodwin [25]. The array spacing is $d_{i}=m_{i} / 2$. The relatively prime integer is $m_{i}$, and the array spacing is $d_{i}$. This method is only suitable for an array spacing with relatively prime integers. The sample antenna array spacings for a three-element array using the presented methods with FOV $|\theta|<\pi / 2$ are given in Table 1 .

4.4. Probability of Ambiguity for Interferometer DF System with Three-Element Arrays. Generally, it is not possible for a DF system to perfectly resolve the angle ambiguity [26]. As shown in Figure 2, if the measurement error is large, DF ambiguity can occur. This can be obtained using simulations for algorithms such as the cosine-function [30], least-squares, and correlation-coefficient methods to estimate the DOA [31]. Another way to obtain the probability of ambiguity is to use the channel pair RMS phase error of Goodwin [25] or the distance between phase difference lines, as in the work of Lee and Woo [32]. The former requires a simulation whenever the array spacing is selected, but the latter can select the array spacing to satisfy the requirement based on the probability of ambiguity. The probability of ambiguity for a three-element interferometer was found by Lee and Woo [32] as follows:

$$
P_{\text {ambiguity }}=\operatorname{erfc}\left(\left(\frac{R}{2 \sqrt{2}}\right) \sqrt{\left(\frac{S}{N}\right)_{x}}\right),
$$

where

$$
\begin{gathered}
\left(\frac{S}{N}\right)_{x}=\left\{\begin{array}{l}
\left(\frac{S}{N}\right)(1-\cos (\alpha) \sin (\alpha)), \text { for End phase } \\
\left(\frac{S}{N}\right)(1+\cos (\alpha) \sin (\alpha)), \text { for Mid phase, }
\end{array}\right. \\
\operatorname{erfc}(z)=\frac{2}{\sqrt{\pi}} \int_{z}^{\infty} e^{-t^{2}} d t, \\
\alpha=\frac{\pi}{2}-\tan \left(\frac{d_{2}}{d_{1}}\right) \text { with } d_{2}>d_{1} .
\end{gathered}
$$

The ambiguity probability found using (5) is illustrated in Figure 4 [32]. In order to decrease the ambiguity probability, a high SNR and large distance between phase difference lines are required. In addition, an end-phase array configuration is better than a midphase configuration.

4.5. Effect of Reflections due to Aircraft Structures. The effect of reflections due to aircraft structures [4] must be considered. When direct path and indirect path signals such as 


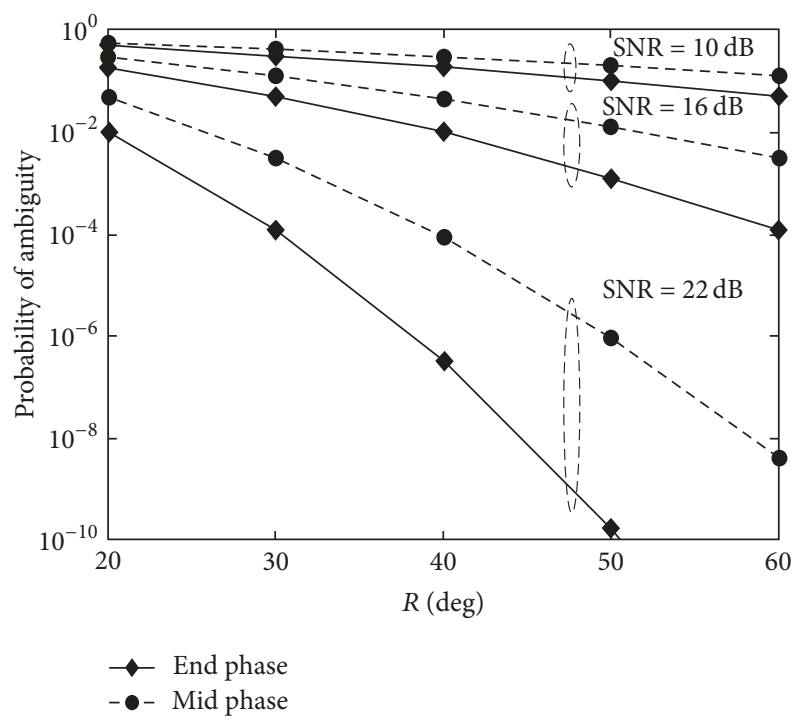

FIGURE 4: Ambiguity probability for three-element interferometer $\left(d_{1}=0.7 \lambda\right.$ and $\left.d_{2}=2.5 \lambda\right)$.

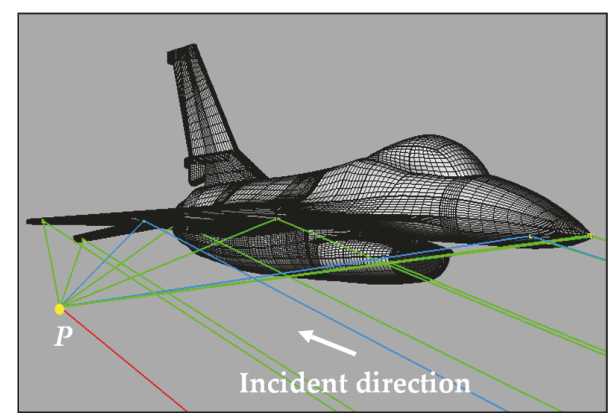

FIGURE 5: CEM model of F-16 fighter aircraft and example of relevant ray-tracing analysis results for receiving point $P$ : red/blue/green lines represent direct/reflected/diffracted rays.

reflections are simultaneously received by an interferometer array, it will measure the vector sum of these signals. If the direct path signal is not sufficiently larger than the indirect path signal, the DF accuracy may be decreased. To minimize the effect of the phase error due to the indirect path signals, the optimum array position throughout the numerical analysis must be selected. Section 5 describes the numerical analysis used to select the array position for ELINT in detail.

\section{Computational Electromagnetic Modeling and Analysis}

Predictions of the performances of the DF antenna array configurations discussed in the previous sections are conducted in an environment where a DF antenna array is isolated from an airborne platform. In that case, two things are assumed. First, all of the antennas in the array operate identically and their radiation patterns are isotropic. Second, direct waves determine the magnitude and phase of the received signals, while reflected or diffracted waves do not exist around the antennas and platform.

In practice, however, the DF antenna array will eventually be installed on a platform such as an aircraft. If the platform is included in the performance prediction, these two assumptions will not be valid, but the following considerations will be necessary. First, the operations of all the antennas in the array should not be the same. Because of the arbitrary shape of the platform and spacing difference between antennas, the ground conditions for different antennas may be different and their radiation patterns and input impedance may also be different from each other [33-35]. Second, reflected and diffracted waves are no longer negligible. A variety of metallic shapes on the platform can cause numerous reflections and diffraction to the direct waves, which results in received signals composed of direct waves and single/multiple reflected and diffracted waves on each antenna. Because these considerations significantly increase the complexity of an analysis of a designed DF system, the performance prediction of the DF antenna array on a platform can be conducted using CEM modeling and analysis [36-38].

CEM modeling is very useful to solve Maxwell's equations governing electromagnetic radiation, scattering, coupling, and so on relative to the corresponding antennas and objects. In order to include as many electromagnetic effects as possible in the problem, the use of full-wave methods is recommended. Various full-wave methods are available, such as the method of moment (MoM), finite element method (FEM), finite discrete time domain method (FDTD) [39], and commercial software products available in the market. Each method has strengths and weaknesses depending on the analysis applications. In the case of open boundary problems like antennas or radar cross section (RCS) analysis, MoM is preferable. On the other hand, if the structure to be analyzed is large enough compared to a wavelength, for example, larger than several tens of wavelengths, a full-wave analysis may be challenging because the computational cost (memory and time) increases significantly. Approximation approaches may be efficient for electrically large modeling problems and physical optics (PO) and the uniform geometrical theory of diffraction (UTD) are typically used as ray-tracing techniques [40].

Figure 5 shows the CEM model of an F-16 fighter aircraft and an example of its relevant UTD-based analysis results for a receiving point $P$. The simulation software AntOpt was used, which was recently developed by the Agency for Defense and Development (ADD) and the Korea Advanced Institute of Science and Technology (KAIST). The CEM model is composed of nonuniform rational B-spline (NURBS) surfaces, which are converted from the 3D CAD data of the aircraft from the Rhino 4 software [41]. All of the surfaces are assumed to be perfect electric conductors when their electromagnetic phenomena are analyzed. If wave signals are incident in the direction shown in the figure, rays arriving at $P$ are obtained from AntOpt. The red, blue, and green lines represent the direct, reflected, and diffracted rays, respectively, and all of the rays contribute to the magnitude and phase of the received electric fields. The results of the received electric fields obtained from AntOpt in blue are 


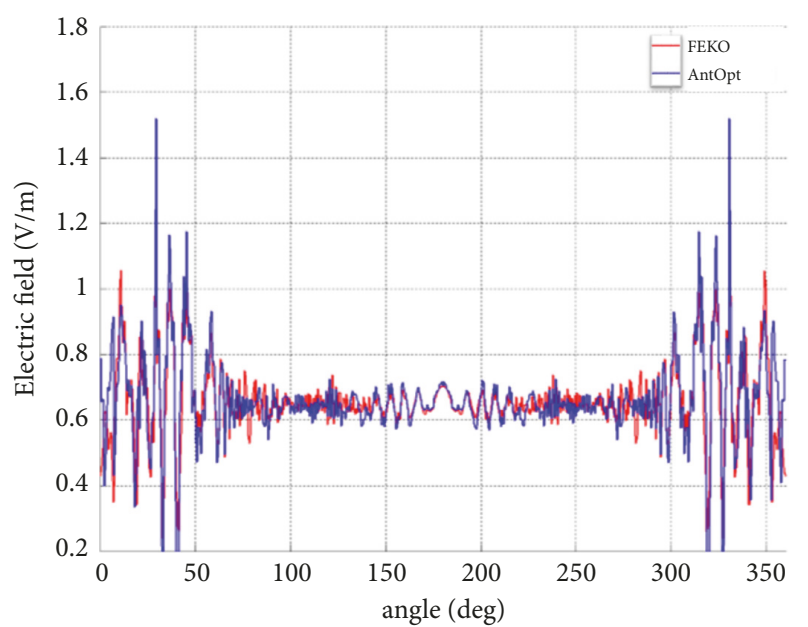

FIGURE 6: Comparison of received electric field strengths computed from commercial software product FEKO (red) and developed software AntOpt (blue) over azimuth angle of signal arrival.

compared to those from the commercial software FEKO [42] based on MoM, as shown in red in Figure 6. The analysis was performed at $2 \mathrm{GHz}$. As shown, a good correlation between the two results was obtained. The computation time consumed by AntOpt and FEKO was approximately $2 \mathrm{~h}$ and $18 \mathrm{~h}$, respectively. Therefore, using the developed AntOpt has the advantages of saving time, even though the software takes into account the electromagnetic effects caused by the aircraft structures.

\section{Conclusion}

This paper presented practical factors that should be considered when designing the array configuration of the DF system for airborne SIGINT. There are many factors to consider when designing the array configuration, but this paper mainly presented antenna consideration factors from a practical point of view, the array configuration methods for airborne COMINT and ELINT and a numerical analysis to select the optimum antenna position.

\section{Conflicts of Interest}

The authors declare that there are no conflicts of interest regarding the publication of this paper.

\section{References}

[1] D. Adamy, EW102: A Second Course in Electronic Warfare, Artech House, Norwood, MA, USA, 2004.

[2] T. Macnamara, Introduction to Antenna Placement And Installation, John Wiley \& Sons, 2010.

[3] H. J. Yang and Y. K. Kwag, "Performance enhancement of direction finding for multiple baseline interferometry," in Proceedings of the 2011 3rd International Asia-Pacific Conference on Synthetic Aperture Radar, APSAR 2011, pp. 1-4, September 2011.

[4] S. Germishuizen and D. C. Baker, "Practical accuracy limitations in airborne microwave interferometric direction finding," in Proceedings of the 1996 4th IEEE AFRICON Conference, pp. 266-271, September 1996.

[5] B. A. Arand, R. Shamsaee, and B. Yektakhah, "Design and fabrication of a broadband blade monopole antenna operating in $30 \mathrm{MHz}-600 \mathrm{MHz}$ frequency band," in Proceedings of the 2013 21st Iranian Conference on Electrical Engineering, ICEE 2013, pp. 1-3, May 2013.

[6] http://www.airport-data.com/aircraft/photo/000552377L.html.

[7] G. W. Stimson, Introduction to Airborne Radar, Scitech Publishing, 2nd edition, 1998.

[8] L. Scorrano, F. Trotta, A. Manna, and L. Dinoi, "Dual-polarization DF Array for airborne SIGINT in VHF / UHF bands," in Proceedings of the 2014 44th European Microwave Conference (EuMC), pp. 1912-1915, October 2014.

[9] T. E. Morgan, "Spiral antennas for ESM," IEEE Proceedings, vol. 132, Pt. F, no. 4, 1985.

[10] S. D. Ahirwar, C. Sairam, and A. Kumar, "Broadband blade monopole antenna covering 100-2000 MHz frequency band," in Proceedings of the Applied Electromagnetics Conference, AEMC 2009 and URSI Commission B Meeting, pp. 1-4, December 2009.

[11] A. Mabrook Masoud and D. Su, "A novel wide-band airborne UHF antenna," in Proceedings of the 2014 IEEE Workshop on Electronics, Computer and Applications, IWECA 2014, pp. 210213, May 2014.

[12] C. Sairam, T. Khumanthem, S. Ahirwar, and S. Singh, "Broadband blade antenna for airborne applications," in Proceedings of the 2011 Annual IEEE India Conference: Engineering Sustainable Solutions, INDICON-2011, pp. 1-4, December 2011.

[13] R. Poisel, Introduction to Communication Electronic Warfare Systems, Artech House, Norwood, MA, USA, 2002.

[14] Environmental Engineering Considerations and Laboratory Test, MIL-STD-810G, 2008.

[15] Environmental Conditions and Test Procedures for Airborne Equipment, RTCA/DO-160G, 2010.

[16] (P.N : MT-2040/E, MT-261002/H) http://www.mtiwe.com/.

[17] S. E. Lipsky, Microwave Passive Direction Finding, SciTech Publishing, 1987.

[18] L. C. Godara, Smart Antennas, CRC Press, 2004.

[19] C. A. Balanis, Antenna Theory, Wiley, 3rd edition, 2005.

[20] L. Osman, I. Sfar, and A. Gharsallah, "An overview of directionof-arrival estimation using an antenna array with four elements," American Journal of Applied Sciences, vol. 9, no. 12, pp. 1979-1984, 2012.

[21] G. Dubost and M. Nicolas, "A Broad Angular Coverage and Large Bandwidth Antenna," in Proceedings of the 27th European Microwave Conference, 1987, pp. 189-194, Rome, Italy, September 1987.

[22] R. O. Schmidt, "Multiple emitter location and signal parameter estimation," IEEE Transactions on Antennas and Propagation, vol. 34, no. 3, pp. 276-280, 1986.

[23] L. Landesa, F. Obelleiro, J. L. Rodríguez, J. A. Rodríguez, F. Ares, and A. G. Pino, "Pattern synthesis of array antennas with additional isolation of near field arbitrary objects," IEEE Electronics Letters, vol. 34, no. 16, pp. 1540-1542, 1998.

[24] C. Lacey, R. Shelor, A. J. Cormier, and F. E. Talke, "Interferometric Measurement of Disk/Slider Spacing: The Effect of Phase Shift on Reflection," IEEE Transactions on Magnetics, vol. 29, no. 6, pp. 3906-3908, 1993.

[25] R. L. Goodwin, "Ambiguity-Resistant Three- and Four-Channel Interferometers," No. NRL-8005, Naval research Lab., Washington, DC, USA, 1976. 
[26] E. Jacobs and E. W. Ralston, "Ambiguity Resolution in Interferometry," IEEE Transactions on Aerospace and Electronic Systems, vol. 17, no. 6, pp. 766-780, 1981.

[27] R. J. McAulay, "Interferometer Design for Elevation Angle Estimation," IEEE Transactions on Aerospace and Electronic Systems, vol. 13, no. 5, pp. 486-503, 1977.

[28] R. G. Wiley, ELINT: The Interception and Analysis of Radar signals, Artech House, Norwood, MA, USA, 2006.

[29] R. McAulay, "Interferometer Design for Elevation Angle Estimation," IEEE Transactions on Aerospace and Electronic Systems, vol. AES-13, no. 5, pp. 486-503, 1977.

[30] C. Park and D. Kim, "The Fast Correlative Interferometer Direction Finder using I/Q Demodulator," in Proceedings of the 2006 Asia-Pacific Conference on Communications, pp. 1-5, Busan, August 2006.

[31] H. Wei and Y. Shi, "Performance analysis and comparison of correlative interferometers for direction finding," in Proceedings of the 2010 10th International Conference on Signal Processing (ICSP 2010), pp. 393-396, Beijing, China, October 2010.

[32] J. H. Lee and J. M. Woo, "The direction finding ambiguity analysis for three element and four element phase interferometer DF system," Journal of the KIMST, vol. 17, no. 5, pp. 544-550, 2014.

[33] A. Leitner and R. D. Spence, "Effect of a circular groundplane on antenna radiation," Journal of Applied Physics, vol. 21, no. 10, pp. 1001-1006, 1950.

[34] F. Obelleiro, L. Landesa, J. M. Taboada, and J. L. Rodríguez, "Synthesis of onboard array antennas including interaction with the mounting platform and mutual coupling effects," IEEE Antennas and Propagation Magazine, vol. 43, no. 2, pp. 76-82, 2001.

[35] A. Kintz and I. J. Gupta, "RF emitter location estimation in the presence of antenna array manifold mismatch," in Proceedings of the Joint 2012 IEEE International Symposium on Antennas and Propagation and USNC-URSI National Radio Science Meeting, APSURSI 2012, Chicago, Ill, USA, July 2012.

[36] A. Bellion, C. Meins, A. J. Vergonjanne, and T. Monediere, "Calibration of direction finding antennas in complex environment," in Proceedings of the 2008 International Union of Radio Science (URSI), Chicago, Ill,USA, 2008.

[37] M. O. Ozec, Direction finding performance of antenna arrays on complex platforms using numerical electromagnetic simulation tools [dissertation thesis], Middle East Technical University, 2011.

[38] A. I. Mackenzie, "EM modeling of far-field radiation patterns for antennas on the GMA-TT UAV," in Proceeding of the SPIE 9460, Airborne Intelligence, Surveillance, Reconnaissance (ISR) Systems and Applications XII, 946009, May 2015.

[39] M. N. O. Sadiku, Numerical Techniques in Electromagnetics, CRC Press, 2nd edition, 2000.

[40] W. L. Stutzman and G. A. Thiele, Antenna Theory and Design, John Wiley \& Sons, 2nd edition, 1998.

[41] Rhino 4.0 user's guide, Rhinoceros, 2008, http://www.rhino3d .com.

[42] FEKO user's manual suite 6.1, EM Software \& Systems - S.A. (Pty) Ltd, 2011, http://www.feko.info. 


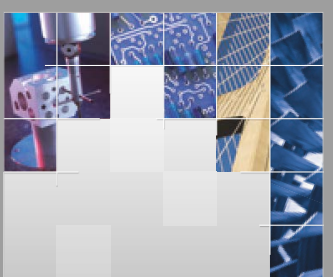

\section{Enfincering}
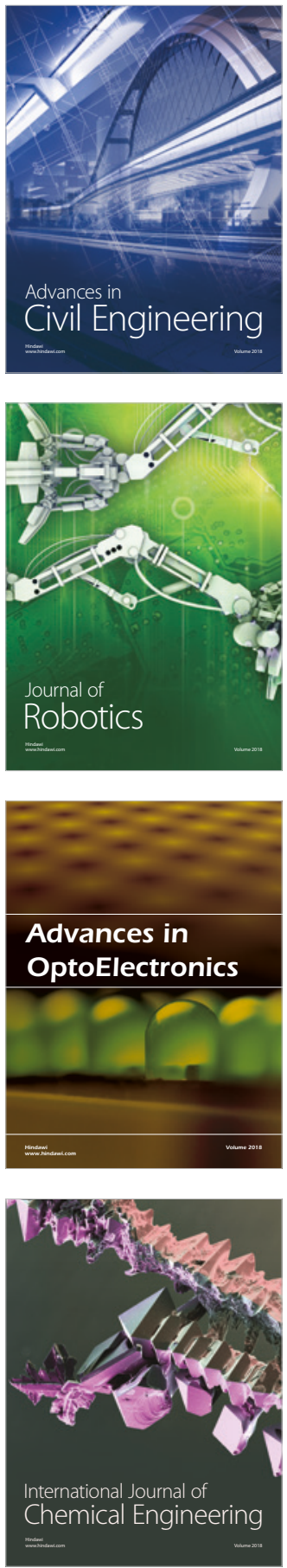

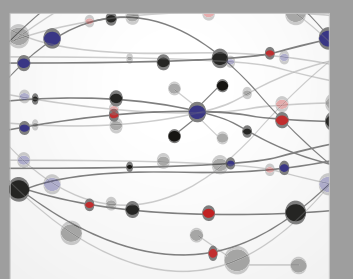

\section{Rotating \\ Machinery}

The Scientific World Journal

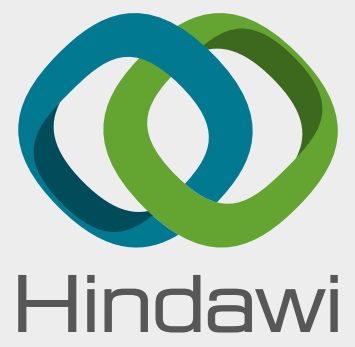

Submit your manuscripts at

www.hindawi.com
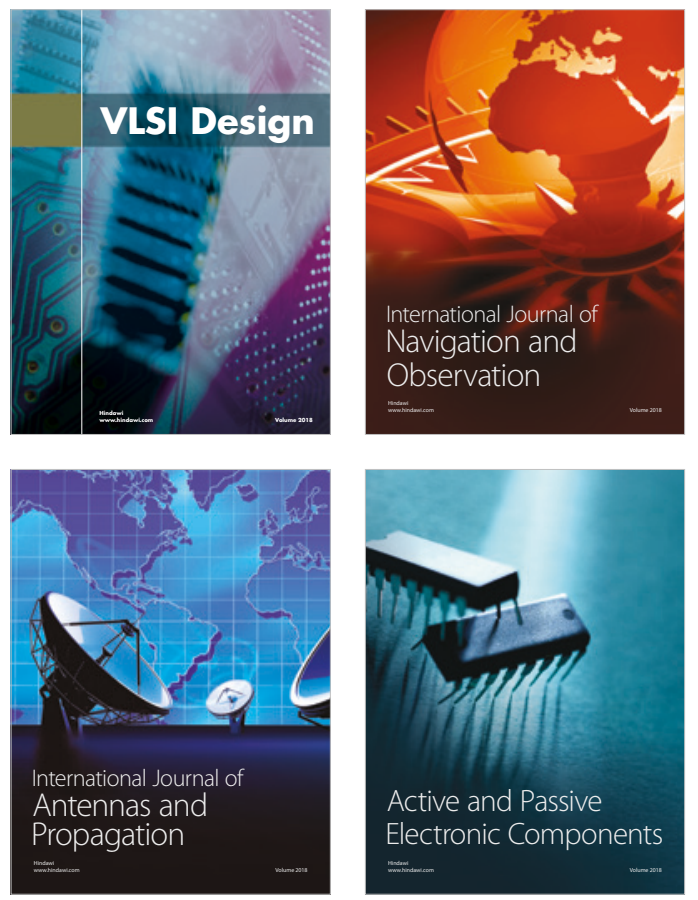
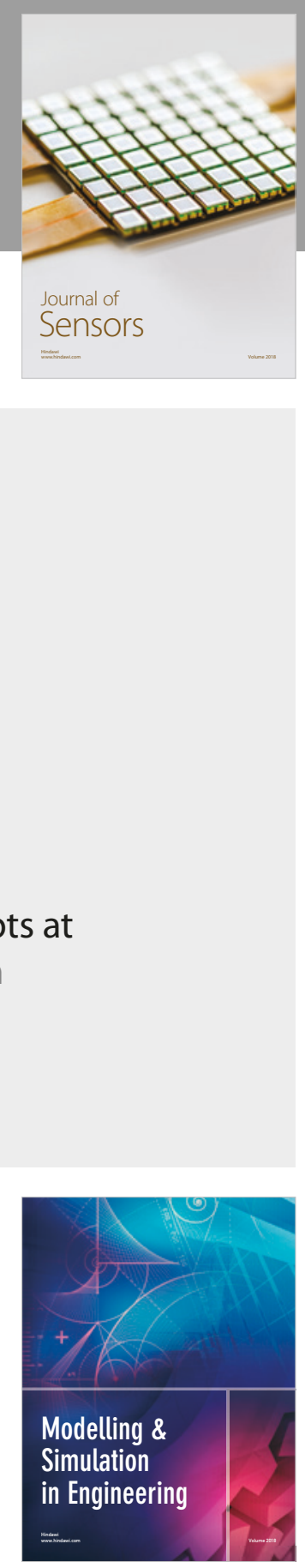

\section{Advances \\ Multimedia}
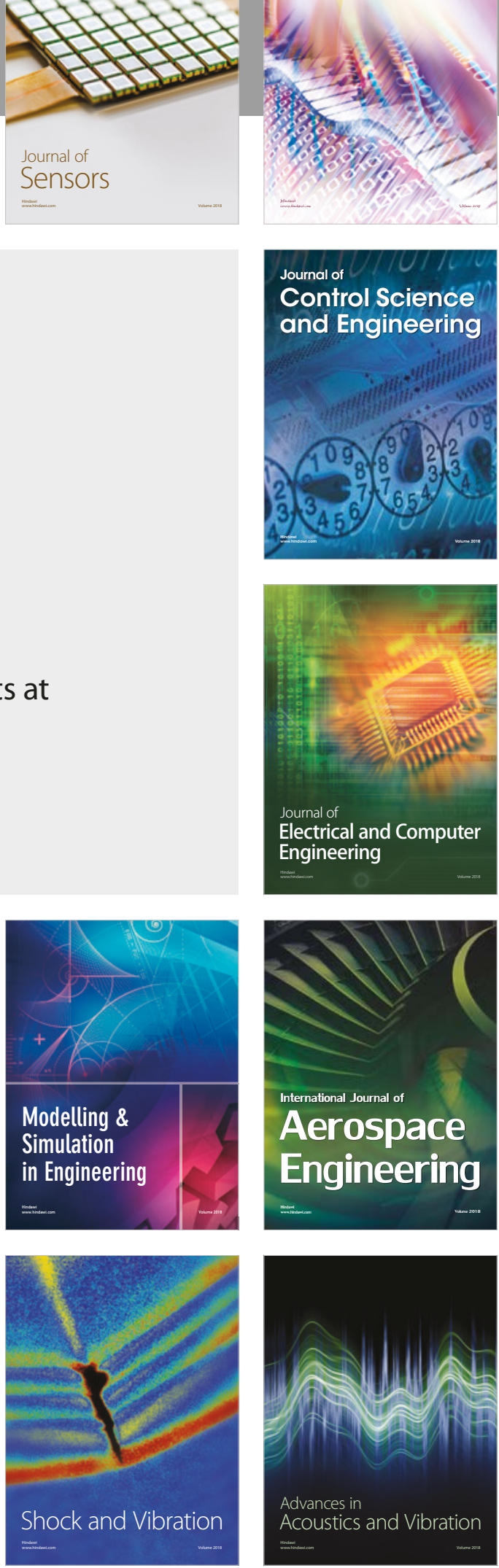